Chair for Management Sciences and Energy Economics University of Duisburg-Essen

EWL Working Paper No. 5

\title{
GAS STORAGE VALUATION UNDER LIMITED MARKET LIQUIDITY: AN APPLICATION IN GERMANY
}

by

Bastian Felix,

Oliver Woll

and

Christoph Weber 


\title{
GAS STORAGE VALUATION UNDER LIMITED MARKET LIQUIDITY: AN APPLICATION IN GERMANY
}

by Bastian Felix, Oliver Woll and Christoph Weber

\begin{abstract}
Natural gas storages may be valuated by applying real options theory. However it is crucial, not to ignore that most evolving gas spot markets, like the German spot market, lack of liquidity. In this context, considering storage operators as price takers does not account for interdependencies of storage operations and market prices. This paper offers a novel approach to storage valuation taking into account the effect of management decisions on market prices. The within this paper proposed methodology determines the optimal production schedule and value by determining the stochastic differential equation describing the storage value and then applying a finite difference scheme. We find that limited liquidity lowers the storage value and reduces withdrawal and injection amounts. Further, we observe decreasing reservation prices for injection and withdrawing for growing illiquidity resulting in a left shift of injection and withdrawing threshold prices.
\end{abstract}

Keywords : natural gas valuation, limited liquidity

JEL-Classification : D52, Q40, Q41

DIPL.-MATH. OEC. BASTIAN FELIX

Chair for Management Sciences and Energy Economics,

University of Duisburg-Essen (Campus Essen)

Universitätsstr. 11, 45117 Essen

++49 - (0)2 01 / 183-2967

www.ewl.wiwi.uni-due.de

bastian.felix@uni-due.de

PROF. DR. CHRISTOPH WEBER

Chair for Management Sciences and Energy Economics, University of Duisburg-Essen (Campus Essen)

Universitätsstr. 11, 45117 Essen

++49 - (0)2 01 / 183-2966

www.ewl.wiwi.uni-due.de

christoph.weber@uni-due.de
DIPL.-KFM. OLIVER WOLL

Chair for Management Sciences and Energy Economics, University of Duisburg-Essen (Campus Essen)

Universitätsstr. 11, 45117 Essen

++49 - (0)2 01 / 183-3389

www.ewl.wiwi.uni-due.de oliver.woll@uni-due.de

The authors are solely responsible for the contents which do not necessarily represent the opinion of the Chair for Management Sciences and Energy Economics. 


\section{Introduction}

Natural gas storages offer flexibility in uncertain markets. The storage owner has the possibility to buy and inject gas, when prices are low and to sell gas, when prices are high. In uncertain markets this flexibility creates an additional value. Recent research has started to examine this value by applying real option theory. To solve this valuation problem De Jong and Walet (2005), Ludkovski and Carmona (2007) and Boogert and de Jong (2008) apply Least Squares Monte Carlo Simulations, first derived by Longstaff and Schwartz (2001) for the valuation of financial options. By contrast Holland (2007) applies a simple Monte Carlo Simulation based approach and Maragos (2002) employs a forward curve simulation to the storage valuation problem. Beside these simulation based methodologies it is possible to determine the optimal management of a storage by deriving a stochastic differential equation and solving this equation analytically (Hodges (2004)) or numerically (Thompson et al. (2003)). Other applications of real options to energy related topics include also the work Tseng and Barz (2002) applying real options to evaluate the flexibility offered by a power plant and Hahn and Dyer (2008), who determine the value to switch between gas and oil extraction, employing a recombining tree approach. However all these approaches assume the asset owner to act as price taker with his decision not affecting market prices. However in many parts of the world, including continental Europe, markets for natural gas are still in their infancy and commonly lack of market liquidity. In this case the management decision of a storage owner and market prices will be interdependent since decisions of an individual storage operator are likely to affect market prices.

The understanding of liquidity is quiet different in literature. Keynes (1930) denotes an asset as liquid if it is "realisable at short notice without loss". According to Amihud and Mendelson (1986) "Illiquidity can be measured by the cost of immediate execution." Geman (2007) states that "Liquidity may be measured by the size of the trade it takes to move the market." In Ghysels and Pereira (2008), "an asset is liquid if large quantities can be traded in a short period of time without moving the price too much". Further definitions of liquidity can be found in Brennan and Subrahmanyam (1996), Vayanos (1998) or Boyle and Guthrie (2003). Conversely, illiquidity may be measured by the impact individual trades have on the market price. This is the definition retained here.

To analyse the impact of this limited market liquidity, we propose an advanced model for storage valuation, incorporating a market liquidity function. The proposed methodology supposes that the storage operator anticipates the limited market liquidity and takes it into the account in each of his operating decisions. This approach may easily be adapted to the valuation of other flexible assets interacting with liquidity-limited markets. 
The remainder of this paper is organized as follows: section 2 analyses liquidity in gas markets and modeling approaches to quantify the possible impact of storage operations to market prices. The third section describes the proposed valuation methodology. Subsequent, the fourth section applies the methodology to an exemplary German storage facility. Finally section 5 concludes.

\section{Market liquidity}

\subsection{Liquidity on natural gas markets}

The most important market places for natural gas in Europe are the NBP (National Balancing Point) in the United Kingdom, the Dutch TTF (Title Trasfer Facility) and the Zeebruegge Hub in Belgium. The NBP, founded in 1996, is the oldest and largest of these markets. It allows trading of natural gas spot and futures contracts. Trading is possible from one day ahead to 10 years ahead. The Zeebrugge Hub, established in the year 2000, is the first hub in Europe providing possibilities of intercontinental gas trading given the nearby LNG terminal. The products range from spot to futures products. The TTF, set up in 2003 , is rather similiar to the NBP and allows also trading of short- and long-term futures.

Table 1 gives an overview of the most relevant data for the three gas markets. It is obvious that the total trading volume at the NBP by more than thirty five times higher than the volume at the TTF and exceeds the volume at the Zeebrugge hub by a factor of more than twenty. The TTF has a lower total trading volume than the Zeebrugge hub. Regarding the national consumption at the NBP the ninefold of the national consumption volume was trated in 2007, whereas the volume traded at the TTF just account for little more than two-thirds of the Dutch consumption. At the Zeebruegge Hub more than twice of the national consumption is traded. Finally the traded volume in Germany accounts for less than a tenth of a percent in 2007. A further important characteristic of a market is the churn rate defined as relation of virtually traded volume to physically traded volume. We calculated the churn rate for the market places above and found that the results are within the range described by Stern (2007).

As reference for a liquid market place commonly the "Henry Hub"', as the largest hub in the world, is cited. This hub is a high liquid natural gas market place with a churn ratio of one hundred. (IEA (2002)) As Table 1 depicts the liquidity of the European markets is substantial below the liquidity of the "'Henry Hub"' market place. As measured by the churn rate the NBP is the market place with the highest liquidity. Although the liquidity of TTF and the Zeebruegge Hub is low, these markets 
Table 1: Key liquidity statistics of major European gas markets.

\begin{tabular}{|c|c|c|c|c|c|c|c|c|}
\hline \multirow[b]{2}{*}{ Year } & \multicolumn{2}{|c|}{$\begin{array}{l}\text { NBP } \\
\text { UK }\end{array}$} & \multicolumn{2}{|c|}{$\begin{array}{c}\text { TTF } \\
\text { Netherlands }\end{array}$} & \multicolumn{2}{|c|}{$\begin{array}{c}\text { Zeebruegge } \\
\text { Belgium }\end{array}$} & \multicolumn{2}{|c|}{$\begin{array}{c}\text { NCG } \\
\text { Germany }\end{array}$} \\
\hline & 2003 & 2007 & 2003 & 2007 & 2003 & 2007 & 2003 & 2007 \\
\hline Traded Volume [TWh] & 6755 & 9955 & 24 & 290 & 430 & 447 & $\ldots$ & $\cdots$ \\
\hline Churn Rate & 12.7 & 14.0 & 1.8 & 3.7 & 3.8 & 5.1 & $\ldots$ & 1.7 \\
\hline $\begin{array}{l}\text { National } \\
\text { Consumption [TWh] }\end{array}$ & 1109 & 1059 & 465 & 430 & 187 & 192 & 1033 & 999 \\
\hline
\end{tabular}

Note: Data source: own calculations based on Gastransportservices (2009), Huberator (2009) and IEA (2008).

offer higher liquidity than the virtual trading point in the German grid area "'NCG"' (Net Connect Germany) installed in 2007. The churn rate of this market was about 1.7 in 2007, (IEA (2008)) which is less than half of the liquidity of the "illiquid"' TTF, allthough trading increases rapidly at the NCG. In January 2007 the amount of trades was about one TWh. Within less than a year this amount expanded to 21 TWh in December 2007. (IEA (2008)) In 2008 NCG stayed the European market place with the highest trading volume growth rates. Whereas the traded volume at the NCG growed by more than 280 percent, the traded volume growth at the other market places was about 120 percent for the TTF, twelve percent for the NBP and six percent for the Zeebruegge Hub. (IEA (2009))

\subsection{Liquidity modelling}

In the literature, several methods for measuring liquidity have been developed. Amihud and Mendelson (1986) base their calculations on the bid-ask spread and focus in the case of stock markets on the the return-spread relation. For modelling the impact of trading volumes on prices, the work of Kempf (1999) as a measure of liquidity is however more suited. He also bases his calculations on the bid-ask spread but provides a regression model of the dependency of prices and volumes. According to Kempf (1999) the regression function is then given by

$$
p(t)-S(t)=\alpha x(t)+\epsilon(t) .
$$

Based on this regression the liquidity is described by the slope $\alpha$ of the corresponding price demand curve:

$$
p(x)=p^{0}-\alpha x
$$


Hence a large $\alpha$ correspondes to an illiquid market, whereas $\alpha=0$ represents the case of a perfect market. The liquidity measure $\alpha$ may depend on time $t$ and trading volume $x$. Due to the lack of empirical data we assume $\alpha$ to be time and volume independent. To measure liquidity for a certain market commonly order book data are necessary. However order books of the relevant markets are in general not published. Thus, recent research considering market liquidity like Hall and Hautsch (2007) deals with limit order book modelling. For our purpose a detailed modelling of the order book is not essential, due to the fact that the operating strategy mostly depends on the resulting price demand function. Figure 1 illustrates the interdependence between order book, bid-ask spread, trading volume and price demand function.

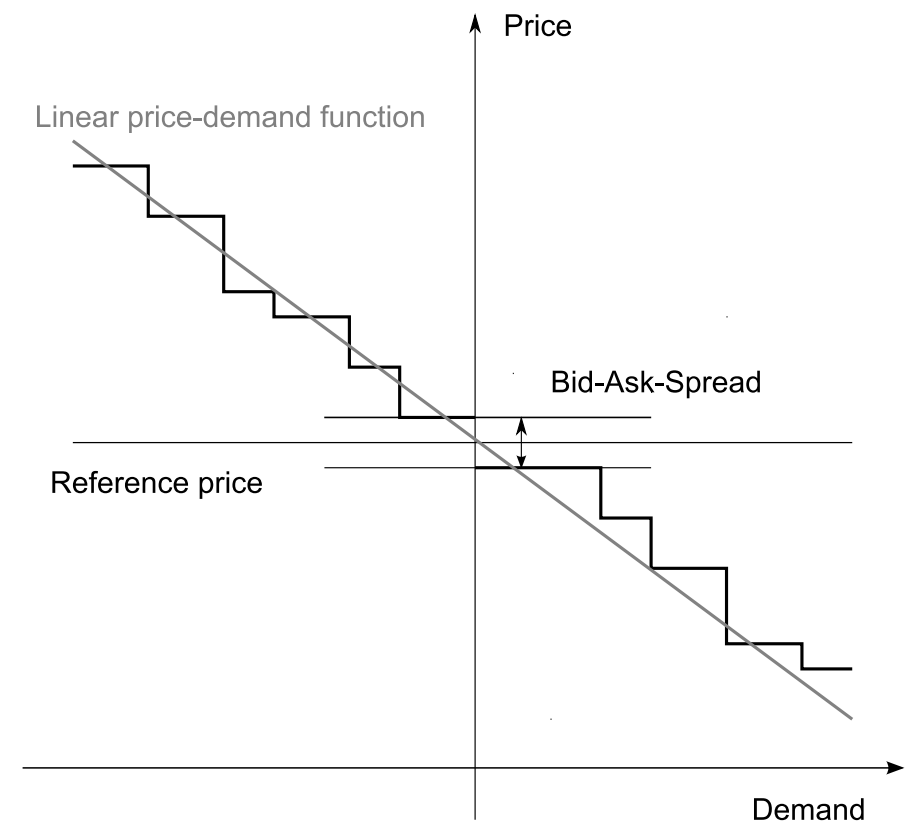

Figure 1: Exemplary order book and liquidity function.

A fast and simple way to evaluate $\alpha$ is taking the average bid-ask spreads and average trading volume to calculate $\alpha$. This estimation is based on bid-ask spreads $S$ and trading volume $x$. Here $S$ is defined as follows:

$$
S=p^{b i d}-p^{a s k} .
$$

Applying the parameters defined above, $\alpha$ is calculated as follows:

$$
\alpha=\frac{\bar{S}}{2 \bar{x}} .
$$

For a better evaluation, a linear regression can provide more information. For the German electricity markets, Weber and Woll (2008) measured the impact of the trading volumes to the electricity 
price applying a price demand function introduced above. They included this liquidity function in a portfolio optimization. In this context the consideration of liquidity shows different results in comparison with other portfolio optimization approaches.

\section{Storage valuation under limited market liquidity}

In evolving deregulated markets with a high degree of uncertainty, assets like natural gas storages offer the possibility to react flexibly to changing market conditions. Nevertheless the value of flexibility is limited by the liquidity of the considered market. Extending the gas storage valuation model of Thompson et al. (2003), we postulate a storage operator who takes the market liquidity into the account of his decision calculus. Under the assumption, the proposed methodology determines the optimal value and operation simultaneously. The option offered by a natural gas storage contains the managerial flexibility offered by other real options. In fact gas storages offer the possibility to buy or sell a good as well as to wait for changing markets conditions. Since these are the operational possibilities offered also by many other operative real options, the proposed methodology may easily be adjusted to other types of real options.

Beside spot market prices and market liquidity, the technical constraints of a storage have a major impact on the value and operation of a storage. Therefore, prior to the mathematical model description, a brief overview on fundamental technical and economic storage characteristics is given in the following.

\subsection{Model formulation}

Underground storage sites may be divided into two main types: on the one hand pore storages, beeing mostly aquifers or former gas fields, and on the other salt cavities. (IGU (2006), FERC (2004)) Whereas former gas fields offer high storage capacities, salt cavities commonly have lower capacities. Stored gas has to be devided into the amount of gas required to deliver the essential technical pressure, labeled cushion or thereby base gas, and the amount of gas actually injected or withdrawn, the working gas. For former gas fields, the share of cushion gas can attain up to fifty percent of the total storage volume. Salt cavities, built by solution mining of salt domes, have lower cushion gas ratios, here up to thirty-five percent of the total storage volume are used as cushion gas. (IEA (1994)) Beside the amount of gas that can be stored the flexibility of a storage facility is basically influenced by the maximum injection and withdrawal rates $c_{\min }$ and $c_{\max }$. Since salt cavities offer high withdrawal and injection rates, they provide higher flexibility and may be cycled on a higher frequency within a year than former gas fields. Withdrawal and injection 
rates are influenced by the pressure within the storage facility and consequently they depend on the storage level $I(t)$. Lower inventory levels permit higher injection levels whereas higher stock levels result in larger withdrawing rates.

Alongside these technical constraints, also the operating costs $a(I, c)$ of a storage facility affect the operation and value of a storage. A major part of the variable costs is determined by the amount of gas used for injecting the gas into the storage respectively withdrawing it. Thus operating costs may be approximated by the rate of gas losses which is commonly taken as one percent of the withdrawn/injected gas. (Dietert and Pursell (2000)) This amount of gas is notably used to run the compressors that inject the gas into the storage or to adjust the temperature and the pressure of the withdrawn gas in order to match the conditions of the connected pipeline grid. Beside the technical constraints of a storage facility, the assumed price process for the underlying commodity, natural gas, has a major impact on the management and the value of the facility. Hence it is necessary to apply an appropriate price model. To cope with a broad class of underlying price processes, the following general jump diffusion process is proposed to describe the price movements:

$$
d P=\mu(P, t) d t+\sigma(P, t) d X+\Phi d q .
$$

Here the deterministic drift rate $\mu(P, t)$ can incorporate also a mean reversion rate. In the second term $d X$ denotes the increment of a Brownian motion. Within the third term, the increments $d_{q}$ of a Poisson process are defined as follows:

$$
d q= \begin{cases}1 & \text { with probability } \lambda(P, t) d t \\ 0 & \text { with probability }(1-\lambda(P, t)) d t\end{cases}
$$

With $\lambda$ denoting the average frequency of a jump per unit of time. This price process allows capturing major characteristics of natural gas prices: seasonal cycles, mean reversion and jump components. Since an important share of European natural gas demand is used for domestic heating, natural gas prices are significantly influenced by temperature and thus exhibit seasonal cycles. Additionally gas storage is only for limited quantities and residential demand is almost price inelastic. Thus high demand facing limited supply can trigger significant price jumps. Periods of extreme weather are however of limited duration and afterwards prices tend to normality - thus mean reversion is to be expected. On the other hand, the proposed price process also encompasses alternative stochastic specifications such as the so-called geometric Brownian motion.

The aim of the model is to assess the value of the flexibility to buy or sell any amount $c(t, P, I, \alpha)$ 
of the underlying good within the given capacity constraints $c_{\min }$ and $c_{\max }$ under consideration of the given price $P$, the storage content $I(t)$ and the given market impact of an action $\alpha$. Taking an interest rate of $\rho$ into account and assuming a scrap value of zero at the end of the valuation period $T$, the objective function at the beginning of the valuation period can be written as follows:

$$
\max _{c(t, P, I, \alpha)} E\left[\int_{0}^{T} e^{-\rho \tau}(c-a(I, c))(P-\alpha c) d \tau\right] .
$$

In an illiquid market, operating the storage facility will have an impact on the price, thus the objective function is calculated taking into account the price demand function including the liquidity parameter $\alpha$. As $\alpha$ is assumed to be non-negative, buying an amount of the underlying good (negative $c$ ) leads to increasing market prices, whereas selling the underlying results in lower prices. Therefore, the amount that is sold or bought is determined under consideration of the market impact of the selected action. To solve this scheduling problem, the value at time step $t$ is analogously formulated as:

$$
V(t, P, I)=\max _{c} E\left[\int_{t}^{T} e^{-\rho(\tau-t)}(c-a(I, c))(P(\tau)-\alpha c) d \tau\right]
$$

Splitting the intervall $[t, T]$ into $[t, t+d t]$ and $[t+d t, T]$, equation (8) can be rewritten as follows:

$$
\begin{aligned}
V(t, P, I) & =\max _{c} E\left[\int_{t}^{t+d t} e^{-\rho(\tau-t)}(c-a(I, c))(P-\alpha c) d \tau\right. \\
& \left.+\int_{t+d t}^{T} e^{-\rho(\tau-t)}(c-a(I, c))(P-\alpha c) d \tau\right] .
\end{aligned}
$$

Using equation (8) the (basic) problem can be reformulated as a dynamic programming problem:

$$
\begin{aligned}
V(t, P, I) & =\max _{c} E\left[\int_{t}^{t+d t} e^{-\rho(\tau-t)}(c-a(I, c))(P-\alpha c) d \tau\right. \\
& \left.+e^{-\rho d t} V(t+d t, P+d P, I+d I)\right] .
\end{aligned}
$$

Whereas the storage volume change $d I$ is calculated as follows:

$$
d I=-(c+a(I, c)) d t
$$

For a sufficiently small time increment $d t$, the first part of equation (10) can be understood as the immediate cash flow resulting from a decision at time step $t$. The second term then represents the expected discounted future value after the decision at time step $t$. Applying a Taylor's Series 
expansion and Itôs Lemma (Øksendal and Sulem (2007)) to equation (10) we get:

$$
\begin{aligned}
V & =\max _{c} E[(c-a(I, c))(P-\alpha c) d t+(1-\rho d t) V \\
& +(1-\rho d t)\left(V_{t}+\frac{1}{2} \sigma^{2} V_{P P}+V_{P} \mu-(c+a(I, c)) V_{I}\right) d t \\
& \left.+(1-\rho d t)\left(\sigma V_{P} d X+\left(V^{+}-V\right) d q\right)\right] .
\end{aligned}
$$

Where $V^{+}=V(t, P+\Phi, I)$ represents the value of the storage if the price has jumped by an amount of $\phi$. Applying Itô calculus, taking expectations and dividing through $d t$ results in:

$$
\begin{aligned}
0 & =\max _{c}\left[\frac{1}{2} \sigma^{2} V_{P P}+V_{t}+V_{P} \mu-(c+a(I, c)) V_{I}\right. \\
& \left.+(c-a(I, c))(P-\alpha c)+\lambda E\left[\left(V^{+}-V\right)\right]-\rho V\right]
\end{aligned}
$$

For an optimization with respect to $c$ it is sufficient to focus on the terms in (13) including $c$ :

$$
\max _{c_{\min } \leq c \leq c_{\max }}\left[-(c+a(I, c)) V_{I}+(c-a(I, c))(P-\alpha c)\right] .
$$

Simplifying this equation results in:

$$
\max _{c_{\min } \leq c \leq c_{\max }} O F(c)
$$

Whereas $O F(c)$ is defined as follows:

$$
O F(c)=\left[-\alpha c^{2}+\left(P+\alpha a(I, c)-V_{I}\right) c-a(I, c)\left(V_{I}+P\right)\right] .
$$

To find the optimal control $c_{\mathrm{opt}}$, which maximizes equation (15) we assume the following cost function:

$$
a(c)= \begin{cases}\beta_{W} c & \text { for } c>0, \beta_{W}>0 \\ -\beta_{I} c & \text { for } c<0, \beta_{I}>0\end{cases}
$$

the objective function can be rewritten as follows:

$$
O F(c)= \begin{cases}-\alpha c^{2}\left(1-\beta_{W}\right)+\left(P\left(1-\beta_{W}\right)-V_{I}\left(1+\beta_{W}\right)\right) c & \text { for } c>0, \\ -\alpha c^{2}\left(1+\beta_{I}\right)+\left(P\left(1+\beta_{I}\right)-V_{I}\left(1-\beta_{I}\right)\right) c & \text { for } c<0 .\end{cases}
$$


By contrast to an optimization without respect to limited liquidity this equation is quadratic with respect to $c$. To derive the optimal operating strategy, we apply a first order condition on the first derivative of equation (18) with respect to $c$. Thus the optimal injection and withdrawing amounts are given by:

$$
\begin{gathered}
c_{W}=\frac{P\left(1-\beta_{W}\right)-V_{I}\left(1+\beta_{W}\right)}{2 \alpha\left(1-\beta_{W}\right)} \\
c_{I}=\frac{P\left(1+\beta_{I}\right)-V_{I}\left(1-\beta_{I}\right)}{2 \alpha\left(1+\beta_{I}\right)} .
\end{gathered}
$$

These optimal withdrawing and injection volumes are additionally restricted by withdrawal and injection capacity constraints. Therefore the withdrawal volume must not exceed the maximal possible withdrawal capacity just as the injection volume must not exceed the injection capacity. For a positive $\alpha$ (which corresponds to an illiquid market) it can be seen that the optimal withdrawing (injection) amount is not of the bang-bang control type, but depending on the level of illiquidity quantified by $\alpha$. As we define $c_{W}$ to be positive and $c_{I}$ to be negative, it is possible to derive the threshold prices for withdrawing and injection by applying this conditions to the equations (19) and (20). For a release of gas the following condition must hold:

$$
P>\frac{V_{I}\left(1+\beta_{W}\right)}{\left(1-\beta_{W}\right)}=: P_{\text {out }}
$$

Analogous the price threshold for an injection can be derived:

$$
P<\frac{V_{I}\left(1-\beta_{I}\right)}{\left(1+\beta_{I}\right)}=: P_{\text {in }}
$$

Hence, this price thresholds are influenced by injection and withdrawing costs $\left(\beta_{W}\right.$ and $\left.\beta_{I}\right)$ and the opportunity costs represented by $V_{I}$. As $V_{I}$ can be construed as the incremental/decremental storage value resulting from an injection/withdrawing of gas, an decreasing $V_{I}$ expedites a withdrawing but postpones an injection of gas. We can obtain the optimal storage strategy with respect to these restrictions als follows:

$$
c_{\mathrm{opt}}=\underset{c \in\left\{c_{W}, 0, c_{I}\right\}}{\arg \max }\{O F(c)\} .
$$

Since the second derivative of equation (18) with respect to $c$ is always negative for positive $\alpha$ and $\beta_{W}$ smaller than one, $c_{\text {opt }}$ certainly is a global maximum for given $V_{I}$ and $P$.

To solve the differential equation (13), border and terminal conditions are necessary. Generally, this edge conditions do not differ from those in Thompson et al. (2003). The first condition can be 
derived from equation (7) where a scrap value of zero is assumed. Hence, the following terminal condition can be stated:

$$
V(T, P, I, c)=0
$$

Next, the first derivatives with respect to $I$ are considered for a full and an empty storage. For a full storage it is not possible to inject more gas, hence the incremental value considering $I$ equals zero. Regarding the storage capacity constraints, the storage cannot be filled above the maximum capacity and cannot be emptied below the volume level zero. Hence, additionally the following boundary conditions must hold:

$$
\begin{array}{ll}
(c+a(I, c)) \geq 0 & \text { for } I=I_{\max } \\
(c+a(I, c)) \leq 0 & \text { for } I=0 .
\end{array}
$$

Finally the following condition must be satisfied for the second derivative with respect to $P$ :

$$
\begin{aligned}
& V_{P P} \longrightarrow 0 \text { for } P \text { large } \\
& V_{P P} \longrightarrow 0 \text { as } P \longrightarrow 0
\end{aligned}
$$

Applying $c_{o p t}$ to equation (13), this differential equation is hyperbolic in I. This can cause spurious oscillations. (Thompson et al. (2003)) Thus we adopt the numerical handling of this problem of Thompson et al. (2003) and apply the slope delimiter described in LeVeque (1999).

\subsection{Real options and liquidity}

The main difference of including limited liquidity into the option valuation is that it leads to a quadratic optimization problem. For a price taker, in each moment of time the operation value is a linear function of injection/withdrawal volume. The slope of this function is determined by the price, the opportunity costs $V_{I}$ and the withdrawing or injection costs (cf. equation (18) for $\alpha$ equal to zero). (cf. Thompson et al. (2003)) In this case the goal is to maximize a linear objective function (OF). Figure 2 shows this case for different combinations of $P$ and $V_{I}$. The optimal solution depends on the slope of the OF. A positive slope in addition to a positive corresponding value of the OF let the right boundary being optimal (cf. $\mathrm{OF}_{1}, \mathrm{OF}_{2}$ ). The case of a negative slope and the value of the OF being positive leads to the left boundary as optimal solution. Furthermore all objective functions cross the origin. Therefore, if the slope changes the sign (cf. $\mathrm{OF}_{1}, \mathrm{OF}_{2}$ ), the optimal solution is "'doing nothing"' since this case corresponds to a negative objective function for 
all possible withdrawing and injection strategies. Overall there are only three possible solutions. These are taking the left boundary (injecting at maximum capacity), taking the right boundary (withdrawing at maximum capacity) or doing nothing.

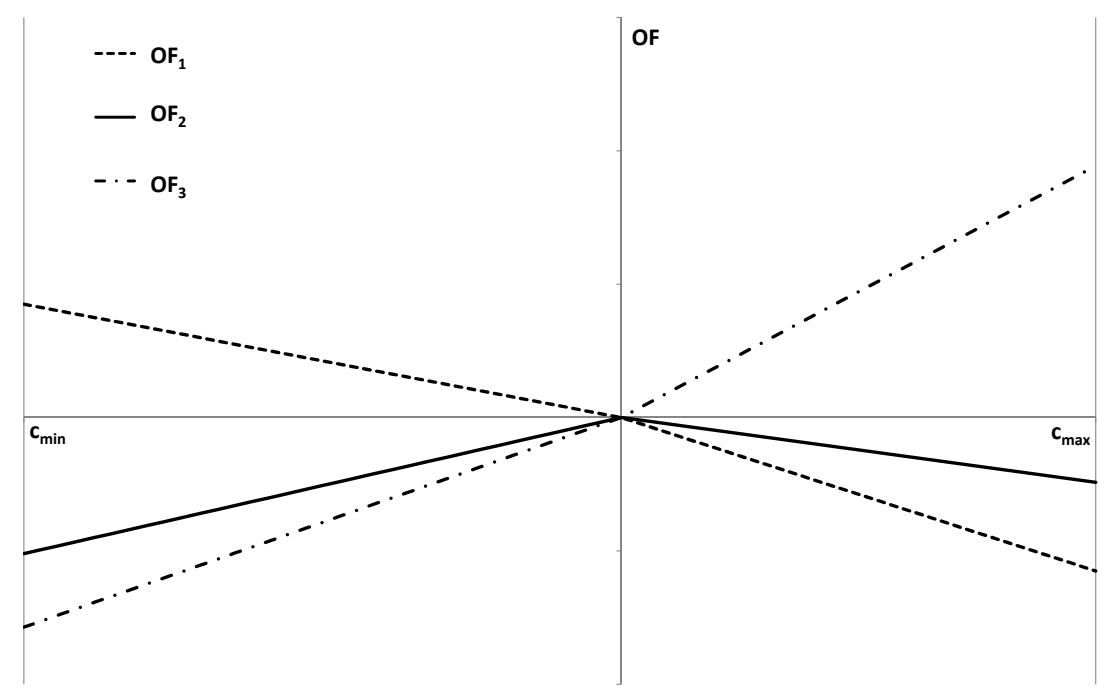

Figure 2: Linear objective function.

In our approach, including liquidity, the goal is to maximize a quadratic OF. Due to the fact that the price is a linear function of injection/withdrawal volume and the operation value is the product of price and injection/withdrawing volume, the $\mathrm{OF}$ is a quadratic function. Figure 3 shows this case. This picture depicts different objective functions depending on the parameter choice of $P$ and $V_{I}$. It is obvious that the set of all maximal turning points is also a quadratic function (solid black line). The optimal solution of our problem depends on the position of the corresponding maximal turning point. As above all objective functions cross the origin. Hence, if the maximal turning point is equal to the origin, the optimal solution is "doing nothing" because it is better to have zero than a negative value as objective (cf. $\mathrm{OF}_{2}$ ). If the maximal turning point is in between the left and the right boundary and positive, the optimal solution results as corresponding value of the maximal turning point (cf. $\mathrm{OF}_{1}$ and $\mathrm{OF}_{3}$ ). If the maximal turning point lies outside of this boundaries, the optimal solution is the corresponding boundary. Thus in our approach every value between the left and the right boundary can arise as optimal solution.

The fact that positive trading volumes (corresponding to withdrawing gas from the storage) have a decreasing effect on the prices, leads to the optimal volume being a piecewise linear increasing function of prices. The non-zero slopes of this function depend on the liquidity of the markets (cf. equations (19) and (20)). The higher the liquidity, the higher is this slope. This is consistent with the linear case, where perfect liquidity is assumed and therefore the non-zero slopes of this 


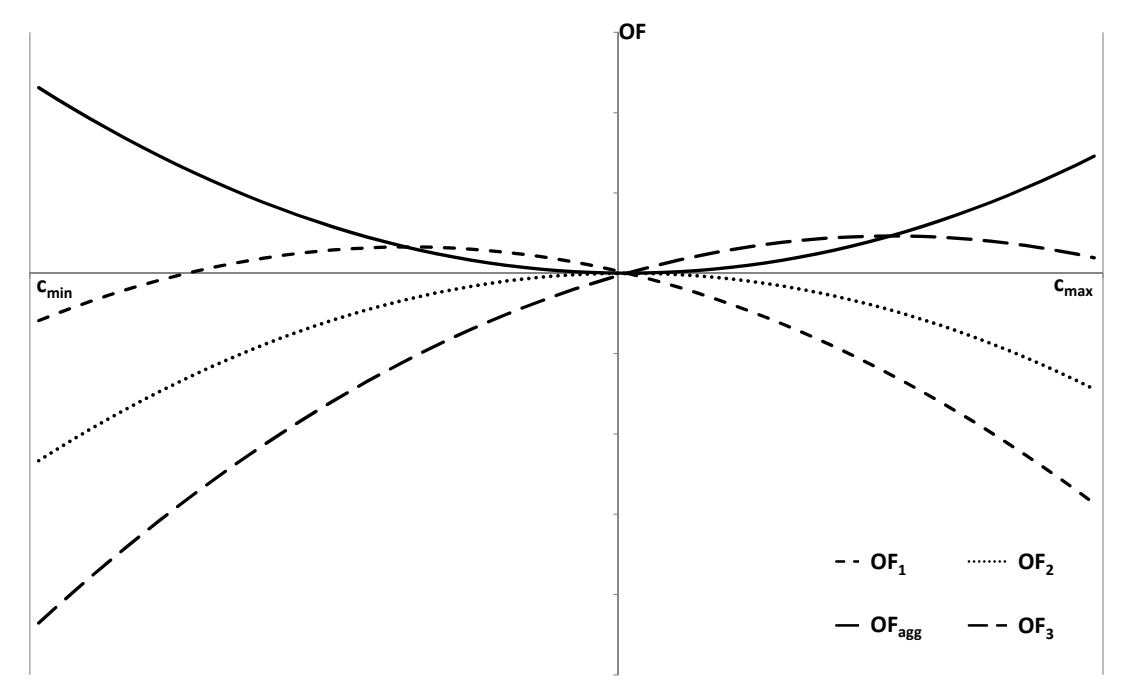

Figure 3: Quadratic objective function.

function are infinity.

Since liquidity can change over time and since different markets show different liquidity, we analyse the dependence of $V$ and $c_{\mathrm{opt}}$ on the liquidity measure $\alpha$. The derivative of $V$ with respect to $\alpha$ results as:

$$
\frac{\partial V}{\partial \alpha}= \begin{cases}-c^{2}\left(1-\beta_{W}\right) & \text { for } c>0 \\ -c^{2}\left(1+\beta_{I}\right) & \text { for } c<0\end{cases}
$$

Since $\beta_{W}$ is defined as fractional amount of $c$, this derivative is always negative. Thus, for decreasing liquidity (increasing $\alpha$ ) the storage value decreases. The derivative of $c_{\mathrm{opt}}$ with respect to $\alpha$ leads to:

$$
\frac{\partial c_{o p t}}{\partial \alpha}= \begin{cases}\frac{V_{I}\left(1+\beta_{W}\right)-P\left(1-\beta_{W}\right)}{2 \alpha^{2}\left(1-\beta_{W}\right)} & \text { for } c_{o p t}=c_{W} \\ 0 & \text { for } c_{o p t}=0 \\ \frac{V_{I}\left(1-\beta_{I}\right)-P\left(1+\beta_{I}\right)}{2 \alpha^{2}\left(1+\beta_{I}\right)} & \text { for } c_{o p t}=c_{I} .\end{cases}
$$

The sign of this derivative depends on the relation of $V_{I}$ and $P$. The case of $\left(V_{I}\left(1+\beta_{W}\right)-P(1-\right.$ $\left.\beta_{W}\right)$ ) being negative, indicates that the derivative is negative and also that the optimal strategy is to withdraw. Hence, an increasing $\alpha$ results in a decreasing withdrawal volume. The opposite way around, if $\left(V_{I}\left(1-\beta_{I}\right)-P\left(1+\beta_{I}\right)\right)$ is positive, the derivative is positive and the optimal strategy is to inject. In this case we have to keep in mind, that injection is defined negative. Therefore an increasing of $\alpha$ leads to an increasing of $c_{\mathrm{opt}}$, what is equivalent to a decreasing injection amount. However, as this is a comparative statics consideration, the generality of these results solely holds for the presumption of $V_{I}$ independent from $\alpha$. 


\section{Application}

In this section the developed approach is applied to determine the optimal schedule and the corresponding revenue of the existing gas storage Epe, owned by E.ON Ruhrgas and located in Germany. This storage offers the possibility to release gas and to sell it at the spot market or to buy at the spot market and inject this gas into the storage. This flexibility is evaluated applying the above derived methodology to the exemplary storage Epe in a market with limited liquidity. Since Epe is located at the border to the Netherlands, data from the Dutch gas exchange, the Title Transfer Facility (TTF) are taken to estimate the liquidity measure and the relevant price process parameters. To illustrate the impact of limited liquidity for the storage valuation, the calculations are done for different levels of liquidity. Thereby a comparison of the optimal schedule and the storage value is done.

\subsection{Storage data}

Employing a conversion factor of $113 \mathrm{~m}^{3}$ for $1 \mathrm{MWh}$ and considering the storage capacity data offered by IGU (2006), Epe offers a withdrawal rate of $18.8 \mathrm{GW}$ and an injection rate of 4.6 GW. Considering the working gas capacity of $13850 \mathrm{GWh}$ it is possible to refill an empty storage within 126 days and to release a full storage in 30 days. Thus the storage can be cycled more than twice in one year. Beside the working gas, the storage must hold a certain amount of cushion gas to keep the essential pressure for the operation of the storage. For Epe this amount is $5585 \mathrm{GWh}$ or 29 percent of the total storage capacity including cushion and working gas.

To cope with volume dependent withdrawal and injection rates, the approach of Thompson et al. (2003), applying the ideal gas law and Bernoulli's equation, is adopted to our specific storage example. We choose this approach due to the comparability to the model of Thompson et al. (2003). Alternative less general but more detailed data, available at the storage operator (E.ON (2007)) can be applied to the methodology to cope with volume dependent injection and withdrawal rates.

Operating costs are approximated according to equation (17). Including transaction and other operating costs beside the losses for injection and withdrawing we assume $\beta_{W}$ and $\beta_{I}$ as two percent.

\subsection{Liquidity estimation}

To compute the liquidity measure $\alpha$ for the regarded gas market, we take day ahead product data from NBP, TTF and Zeebruegge. This data is published on a daily basis and prepares the necessary information for measuring $\alpha$. The available period ranges from 19th february 2007 
to 5th february 2009. The results of applying equation (4) to the historical data $(\bar{S}$ and $\bar{x})$ are displayed in Table 2.

Table 2: Results for liquidity measure $\alpha$.

\begin{tabular}{lccc}
\hline & NBP & TTF & Zeebruegge \\
\hline Bid ask spread $\bar{S}[$ EUR/MWh] & 1.17 & 1.06 & 0.40 \\
Average trading volume $\bar{x}[\mathrm{MW}]$ & 5232.49 & 3477.32 & 927.11 \\
$\alpha$ [EUR/MWh/MW] & 0.00011 & 0.00015 & 0.00021 \\
\hline
\end{tabular}

Note: Data source: own calculations based on Spectron (2008) and Energate (2008).

It is obvious, that NBP is the market with highest liquidity. Although the bid ask spread of Zeebruegge is less than half of the spread of NBP, the liquidity of NBP is nearly twice the liquidity of Zeebruegge. The reason for this is that the average day ahead trading volume of NBP is more than 5 times the volume of Zeebruegge. The liquidity of TTF is in the middle of Zeebruegge and NBP liquidty. Figure 4 depicts historical price and volume data from the TTF. Considering the maximal withdrawal and injection rates of Epe with respect to the TTF market liquidity a withdrawal (injection) of gas at maximum capacity decreases (increases) the market price by $2.82(0.68)$ EUR/MWh. For 2007 this is a decrease of almost twenty percent of the average market price for withdrawing respectively an increase of five percent for injecting.
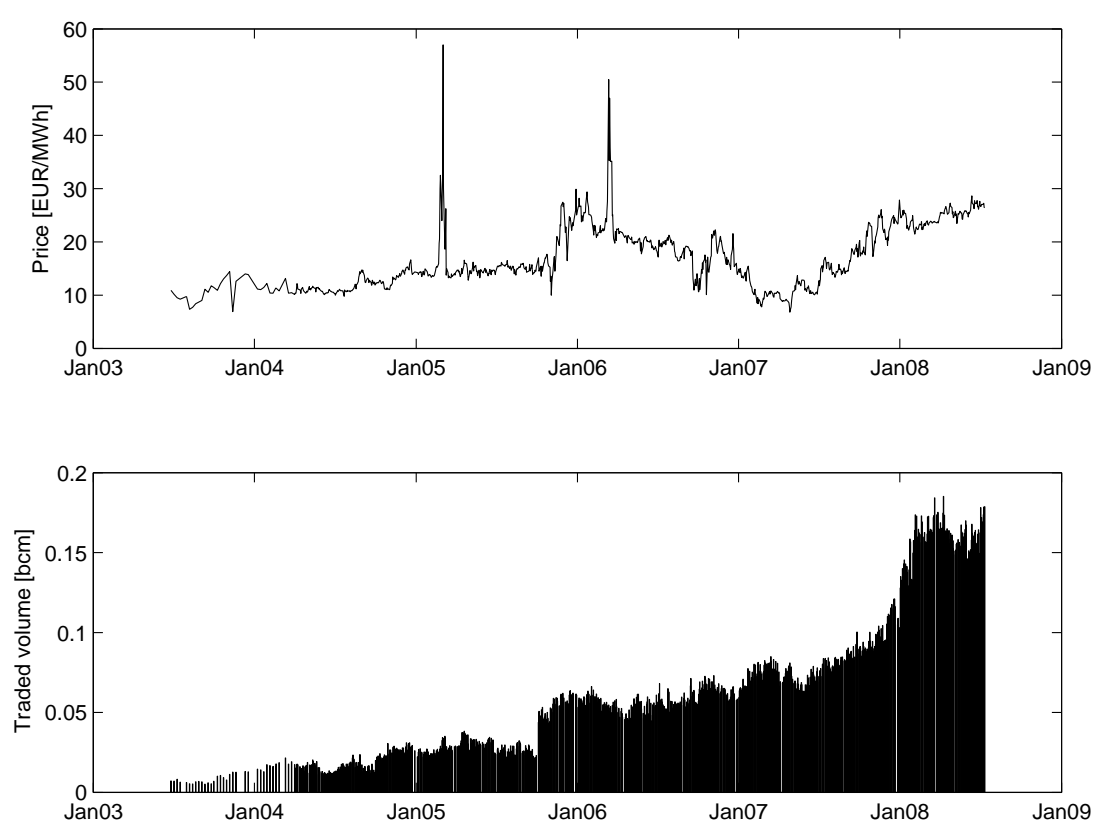

Figure 4: Prices and volumes at TTF. 


\subsection{Price process parameter estimation}

In addition to the storage capacity data and the computation of $\alpha$ it is necessary to compute the relevant parameters of the underlying price process. To incorporate the main characteristics of natural gas prices: seasonality and jump components (cf. Figure 4 ), a mean reversion jump diffusion process is assumed as underlying price process. The parameter estimation is done in two steps: At first the jumps within the historical price data are filtered, applying the approach described in Weron (2006). This approach estimates the jump intensity $\lambda$, the mean jump size $\mu_{s}$ and the jump size standard deviation $\sigma_{s}$ by selecting all historical prices as jumps which are above two standard deviations of the mean price. Subsequent the filtered jumps are used to calculate the relevant jump parameters. This estimation is done iterative until the jump intensity $\lambda$ does not change within a predefined confidence interval. In a second step the parameters of the meanreversion process are estimated applying a least squares regression approach proposed in Dixit and Pindyck (1994) to the adjusted historical data without jumps. For historical price data $P_{t}$ and an error of $\epsilon_{t}$ this is done as follows:

$$
P_{t}-P_{t-1}=A+B P_{t-1}+\epsilon_{t} .
$$

Whereas $A$ and $B$ are the axis intercept and the slope of the regression line. Alternatively to the regression approach a maximum likelihood estimation can be applied. The price parameters were estimated applying the historical spot price index of the TTF in between 2004/04/01 and $2007 / 12 / 31$. Thus an mean reverting level $\theta$ of $18 \mathrm{EUR} / \mathrm{MWh}$ and a mean reversion rate $\kappa$ of 0.005 are resulting. This implies a half life time of 200 days. For the normal distributed jump size $\Phi$ we estimated a mean $\mu_{s}=0$ and a standard deviation of $\sigma_{s}=7.4$. Hence the jump size $\Phi$ is $\mathcal{N}(0,54.7)$ distributed. Calculating the volatility as standard deviation of the filtered absolute price differences as 0.68 and setting $\mu(P, t)=\kappa(\theta-P)$ the used price process can be written as follows:

$$
d P=0.005(18-P) d t+0.68 d X+\Phi d q .
$$

Whereas $d X$ denotes the increment of a standard Brownian Motion as in equation (5). The time span $d t$ is measured in days and $d q$ denotes the increment of a Poisson process with jump intensity $\lambda=0.07$. The storage value is calculated applying a risk free interest rate of ten percent. 


\subsection{Results}

Implementing a simple explicit difference scheme we solve the stochastic differential equation backwards starting at the last valuation day of 262 trading days. Computing the value of the storage it is necessary to choose the discretization sensitivity of $d t$ and $d P$ with respect to each other to achieve numerically stability. (Benker (2005)) Adopting the approach of Thompson et al. (2004) the integral caused by the normal distributed jump size is solved approximated with the trapezoid rule. Time is measured in days. To guarantee numerical stability we applied a time step size of $d t=\frac{1}{8}$ and a price step size of $d P=0.5$. As described in Benker (2005) the following condition for numerical stability of the solution then holds:

$$
d t \leq \frac{d P^{2}}{2}
$$

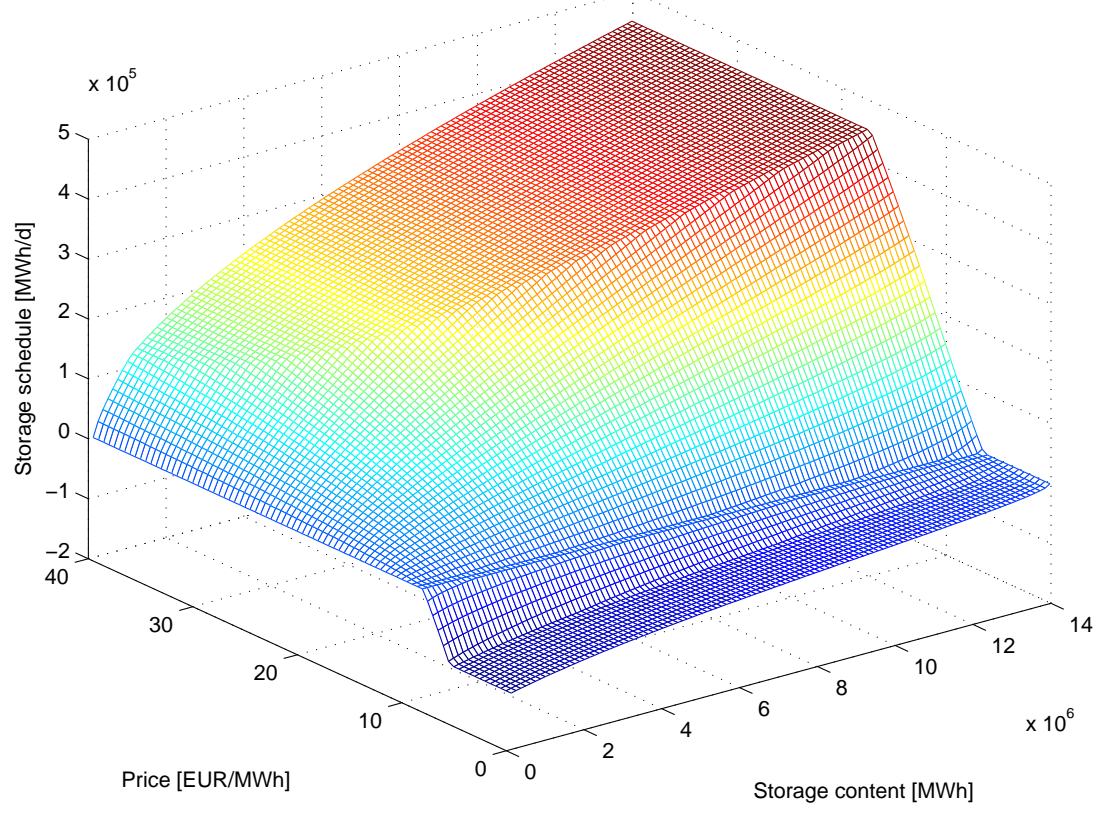

Figure 5: Storage schedule in $t_{0}$.

Figure 5 depicts the resulting storage strategy at the beginning of the valuation period (for $\alpha=$ 0.0002). Furthermore there are three regions visible: injection (negative strategy), "doing nothing" and withdrawing (positive strategy) whereas the maximum injection/withdrawal capacity is affected by the volume of gas within the storage. Within the injection and withdrawal regions it is apparent that the storage operator selects the maximum injection/withdrawal capacity only for very low/high prices. The operating strategies above/below these prices are adjusted due to the price impact 
caused by the limited market liquidity. In contrast to this operating strategy, the storage strategy in $t_{0}$ with perfect liquidity leads to decisions of the bang-bang type: For given thresholds $P_{\text {out }}$ and $P_{\text {in }}$ the strategy for all prices above $P_{\text {out }}$ is to withdraw as much as possible. For all prices below $P_{\text {in }}$ the strategy is to inject as much as possible.
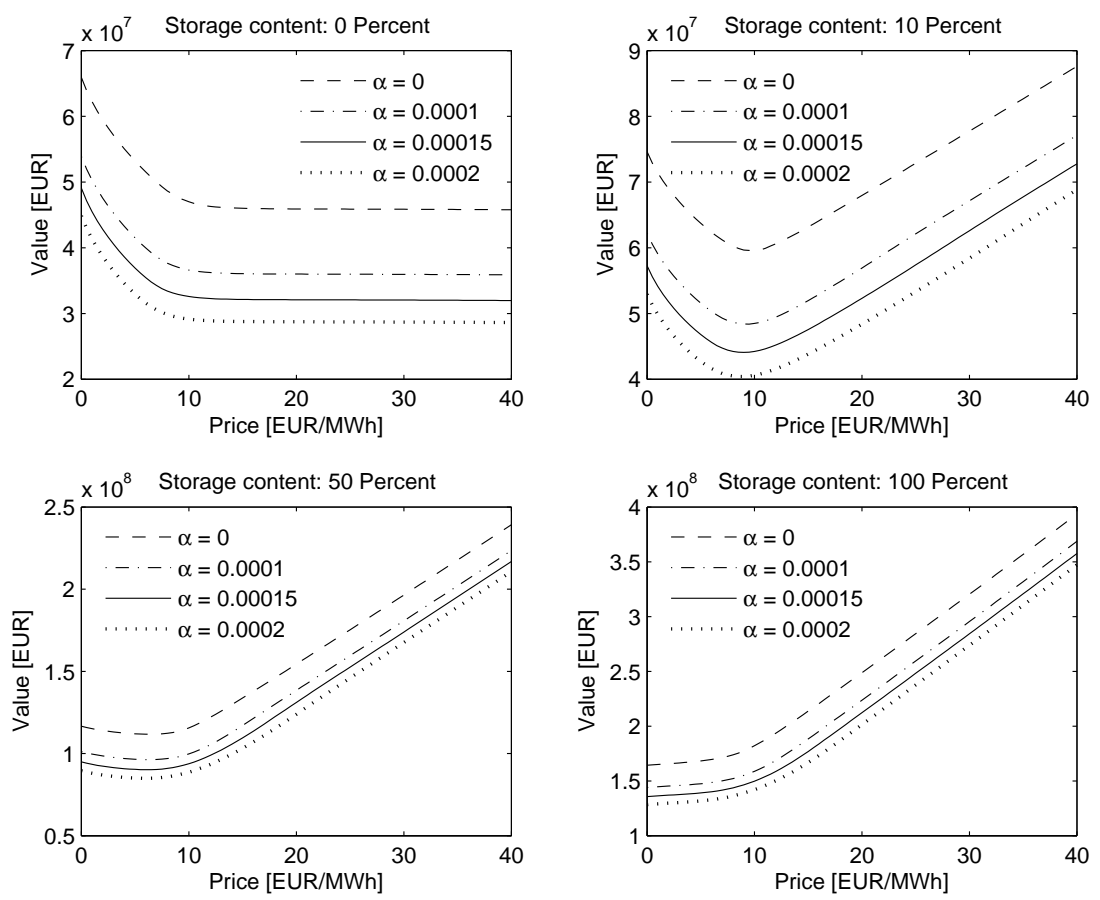

Figure 6: Gas storage value comparison for different storage content levels and different levels of liquidity at time step $t_{0}$.

Figure 6 compares the value of the storage at the beginning of the valuation period for different levels of illiquidity and for perfect liquidity for different volume levels. As Figure 6 depicts, an empty storage at the beginning of the valuation period $t_{0}$ can be seen as a put option on the storage capacity with strike price $V_{I}$. The storage operator has the opportunity, to buy one unit of gas and inject this unit into the storage. As the storage operator injects one unit of gas into the storage, he looses one unit of "flexibility" delivered by the storage. Hence by buying and injecting one unit of gas, the storage operator sells one unit of storage flexibility. On the other hand he obtains the option to sell this gas, when prices are high enough. Hence the value of the above mentioned put option grows with decreasing gas prices. Figure 6 illustrates further, that in an illiquid market the strike price of this option is lower than in a market with high liquidity. In an illiquid market buying one unit of gas increases the market price (cf. equation (29)). Therefore the storage operator delays his decision to inject to lower market prices. As the storage level increases the above mentioned put option turns into a straddle. As for the value of an empty storage, the value of the straddle decreases with increasing illiquidity. A filled storage can be construed as call option. 
In an illiquid market every action of the storage operator affects the market price. This leads to lower values than in markets with perfect liquidity, for all possible volume levels. Thus, postulating a perfect market and assuming the storage operator as price taker can lead to an overestimation of the storage value. Thus, beside the restrictions of withdrawal and injection rates the value of a storage is affected by the level of market liquidity.

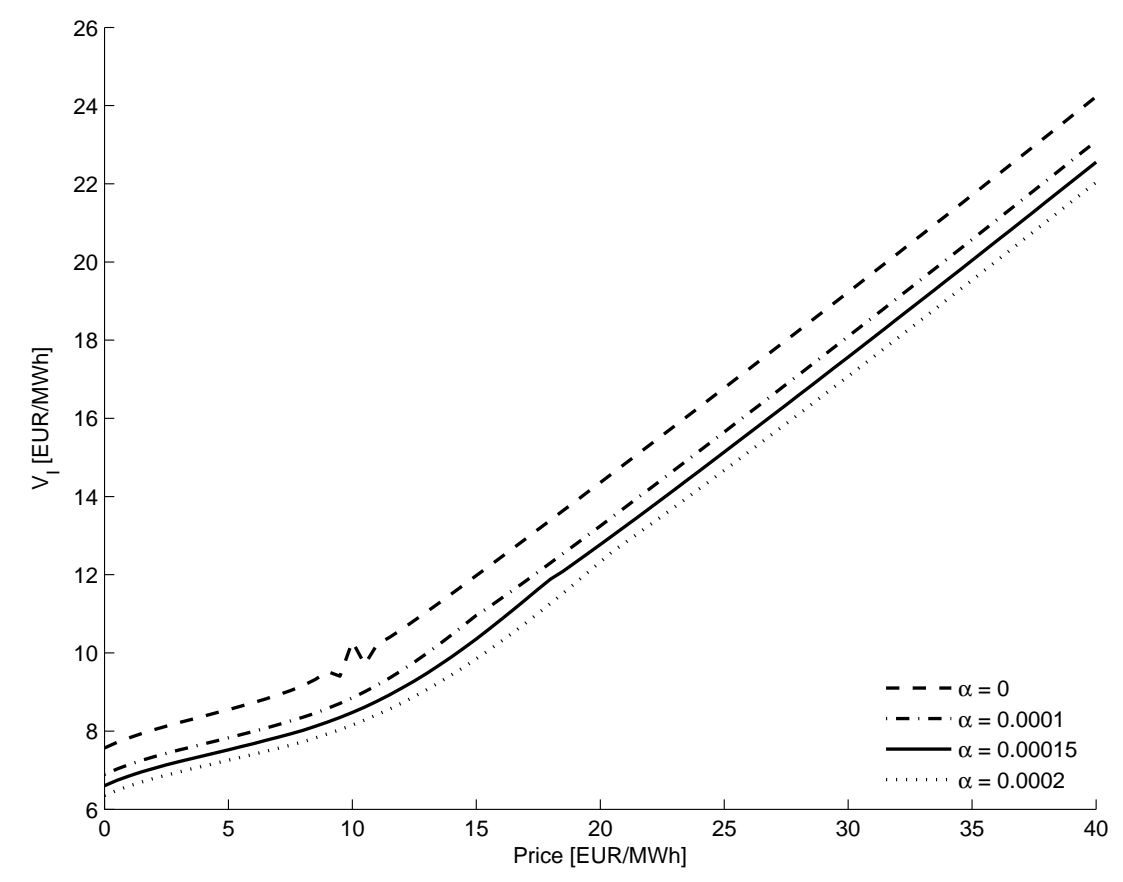

Figure 7: $V_{I}$ sensitivity with respect to $\alpha$ for a storage content of fifty percent at time step $t_{0}$.

Figure 7 shows the sensitivity of the "'strike price"', of the above described options, with respect to $\alpha$ for a half full storage at the beginning of the valuation period. As liquidity decreases strike prices $V_{I}$ (which can also be construed as reservation prices for one unit of gas in the storage) are decreasing. Thus, expected future illiquidity costs, resulting from lower withdrawal returns and higher injection costs, reduce reservation prices. As strike prices vary with respect to $\alpha$, each level of liquidity results in a different option with distinct strike prices and exercise strategies.

Figure 8 depicts the liquidity impact on the storage strategy for a storage level of fifty percent. It can be seen that increasing liquidity (measured by $\alpha$ ) leads to significant higher withdrawal and injection volumes (outside of the "'doing-nothing"' interval of the perfect liquidity strategy). In this case enhancing liquidity by twenty five percent from $\alpha=0.0002$ to $\alpha=0.00015$, which corresponds to the liquidity function difference in between Zeebruegge and TTF, expands the withdrawal rate by almost 20 percent for the mean reversion level of 18 EUR/MWh. Furthermore, higher illiquidity results in a left shift of the price interval where the storage operator whether withdraws nor in- 


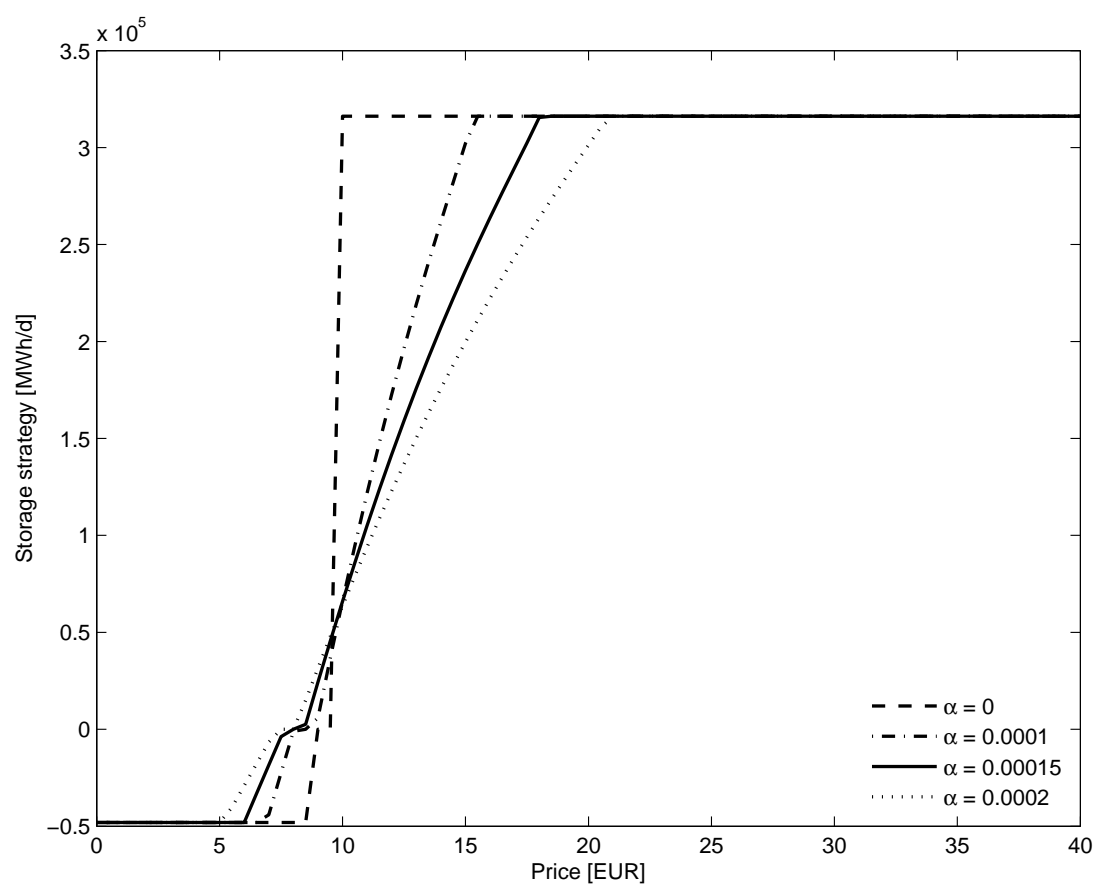

Figure 8: Strategy sensitivity with respect to $\alpha$ for a storage content of fifty percent at time step $t_{0}$.

jects gas into the storage. This corresponds to the price thresholds for injection and withdrawing, derived in the equations (22) and (21), in conjunction with decreasing reservation prices as illustrated by figure 7 . Thus, as decreasing liquidity reduces the value of an in-/decremental unit in the storage, the storage operator waits for lower prices for an injection and thus postpones the injection. On the other hand, the storage operator tends to withdraw earlier as the reservation price decreases. Nevertheless the threshold price for withdrawing at maximum capacity is increasing with increasing illiquidity. Thus for increasing $\alpha$ the storage operator withdraws within a broader price interval below maximum capacity.

\section{Conclusions}

This paper has derived a new methodology to valuate the flexibility of natural gas storages in illiquid markets. This approach can be easily adapted to other assets offering flexibility. We have shown that the existence of an illiquid market decreases the storage value. Additionally the storage strategy has to be adjusted in illiquid markets. We find that increasing illiquidity left shifts the price interval where the storage operator whether injects not withdraws. Furthermore, the operating strategies are reduced in a market with higher illiquidity (outside the price interval where the storage operator would whether inject nor withdraw at perfect liquidity). Future research can also cope with time and volume dependent liquidity measures. The model can be evaluated 
considering different price processes (e.g. incorporating the seasonality of gas prices) or solve the differential equation with a different numerical method, e.g. an implicit finite difference scheme. The focus of future research can also lie on the measurement of the liquidity risk resulting from an illiquid market. Finally, under certain restrictions, the analytical solution of this problem can be of interest.

\section{Acknowledgements}

We thank Istvan Vajda for helpful comments on a draft version of this paper.

\section{References}

Amihud, Y. and H. Mendelson. 1986. Asset pricing and the bid-ask spread. Journal of Financial Economics 17(2), 223-249.

Benker, H. 2005. Differentialgleichungen mit MATHCAD und MATLAB. Berlin, Heidelberg: Springer-Verlag Berlin Heidelberg.

Boogert, A. and C. de Jong. 2008. Gas storage valuation using a monte carlo method. The journal of derivatives 15(3), 81-98.

Boyle, G. W. and G. A. Guthrie. 2003. Investment, uncertainty, and liquidity. Journal of Finance 58(5), 2143-2166.

Brennan, M. J. and A. Subrahmanyam. 1996. Market microstructure and asset pricing: on the compensation for illiquidity in stock returns. Journal of financial economics 41(3), 441-464.

De Jong, C. and K. Walet. 2005. Gas storage management. In V. Kaminski (Ed.), Managing Energy Price Risk (3 ed.)., pp. 631-648. London: Risk Books.

Dietert, J. and D. Pursell. 2000. Underground natural gas storage. http://www.worldoil.com/WO_RESEARCH/Research/062800storage.pdf.

Dixit, A. K. and R. S. Pindyck. 1994. Investment under uncertainty. Princeton, N.J.: Princeton University Press.

Energate 2008. www.energate.de.

E.ON 2007. E.ON Ruhrgas AG. Wesentliche Inhalte eines Speichervertrages. http://www.eon-ruhrgas.com/cps/rde/xchg/SID-3F57EEF5-C59BF9E8/er-corporate/hs.xsl/1584.htm.

FERC 2004. Federal Energy Regulatory Commission. Current state of issues concerning underground natural gas storage. http://www.ferc.gov/eventcalendar/files/20041020081349-final-gs-report.pdf.

Gastransportservices 2009. TTF volume development. http://www.gastransportservices.nl/content/documents/701908/557747.xls. 
Geman, H. 2007. Commodities and commodity derivatives: modeling and pricing for agriculturals, metals and energy (Repr. ed.). Wiley finance series. Chichester [u.a.]: Wiley \& Sons.

Ghysels, E. and J. P. Pereira. 2008. Liquidity and conditional portfolio choice: A nonparametric investigation. Journal of Empirical Finance 15(4), 679-699.

Hahn, W. and J. Dyer. 2008. Discrete time modeling of mean-reverting stochastic processes for real option valuation. European Journal of Operational Research 184(2), 534-548.

Hall, A. D. and N. Hautsch. 2007. Modelling the buy and sell intensity in a limit order book market. Journal of financial markets.

Hodges, S. D. 2004. The value of a storage facility. working paper. Warwick Business School.

Holland, A. 2007. Optimization of injection/withdrawal schedules for natural gas storage facilities. In Twenty-seventh SGAI International Conference on Artificial Intelligence (AI-2007), Cambridge, England. http://4c.ucc.ie/ aholland/publications/GasStorage.pdf.

Huberator 2009. Zeebrugge Hub Monthly Report. http://www.huberator.com/uploadedfiles/documentation/y2000nthly

IEA 1994. International Energy Agency. Natural gas transportation: organisation and regulation. Paris.

IEA 2002. International Energy Agency. Flexibility in natural gas: supply and demand. Paris.

IEA 2008. International Energy Agency. Natrual Gas Market Review: Optimising investments and ensuring security in a high-priced environment. Paris.

IEA 2009. International Energy Agency. Natural Gas Market Review. Paris.

IGU 2006. International Gas Union. UGS Data Bank. http://www.igu.org/html/wgc2006/WOC2database/index.htm.

Kempf, A. 1999. Wertpapierliquidität und Wertpapierpreise. Beiträge zur betriebswirtschaftlichen Forschung. Wiesbaden: Dt. Univ.-Verl. [u.a.].

Keynes, J. M. 1930. A treatise on money: Vol. 2, The applied theory of money.

LeVeque, R. J. 1999. Numerical methods for conservation laws (2. ed.). Lectures in mathematics. Basel [u.a.]: Birkhäuser.

Longstaff, F. and E. Schwartz. 2001. Valuing american options by simulation: A simple least-squares approach. Review Of Financial Studies 14(1), 113-147.

Ludkovski, M. and R. Carmona. 2007. Valuation of energy storage: An optimal switching approach. http://www.pstat.ucsb.edu/faculty/ludkovski/storage-CL.pdf.

Maragos, S. 2002. Valuation of the operational flexibility of natural gas storage reservoirs. In E. I. Ronn (Ed.), Real options and energy management, pp. 431-456. London: Risk Books.

Øksendal, B. K. and A. Sulem. 2007. Applied stochastic control of jump diffusions (2 ed.).

Spectron 2008. http://www.spectrongroup.com/ftpspectrondownload/Historicallndex.xls. 
Stern, J. P. 2007. Is there a rationale for the continuing link to oil product prices in continental european long-term gas contracts? Oxford Institute for Energy Studies.

https://www.oxfordenergy.org/pdfs/NG19.pdf.

Thompson, M., M. Davison, and H. Rasmussen. 2003. Natural gas storage valuation and optimization: A real options application. http://www.apmaths.uwo.ca/mdavison/_library/preprints/Gasstorage.pdf.

Thompson, M., M. Davison, and H. Rasmussen. 2004. Valuation and optimal operation of electric power plants in competitive markets. Operations Research 52(4), 546.

Tseng, C. L. and G. Barz. 2002. Short-term generation asset valuation: A real options approach. Operations Research 50(2), 297-310.

Vayanos, D. 1998. Transaction costs and asset prices: A dynamic equilibrium model. Review Of Financial Studies 11(1), 1-58.

Weber, C. and O. Woll. 2008. Portfolio optimization in electricity trading with limited liquidity.

Weron, R. 2006. Modeling and Forecasting Electricity Loads and Prices: A Statistical Approach. 